\title{
RATIONAL NODAL CURVES WITH NO SMOOTH WEIERSTRASS POINTS
}

\author{
ARNALDO GARCIA AND R. F. LAX
}

(Communicated by Eric Friedlander)

\begin{abstract}
Let $X$ denote the rational curve with $n+1$ nodes obtained from the Riemann sphere by identifying 0 with $\infty$ and $\zeta^{j}$ with $-\zeta^{j}$ for $j=0,1, \ldots, n-1$, where $\zeta$ is a primitive $(2 n)$ th root of unity. We show that if $n$ is even, then $X$ has no smooth Weierstrass points, while if $n$ is odd, then $X$ has $2 n$ smooth Weierstrass points.
\end{abstract}

C. Widland [14] showed that the rational curve with three nodes obtained from $\mathbf{P}_{\mathbf{C}}^{1}$ by identifying 0 with $\infty, 1$ with -1 , and $i$ with $-i$ has no smooth Weierstrass points. This curve may be realized as the projective plane curve $x^{2} y^{2}+y^{2} z^{2}=$ $x^{2} z^{2}$, which has "biflecnodes" at the points $(1,0,0),(0,1,0)$, and $(0,0,1)$, with each biflecnode being a Weierstrass point of weight 8 . In this note, we show that this curve is one member of an infinite family of rational nodal curves with no smooth Weierstrass points. We remark that a general rational nodal curve with $n$ nodes has $n(n-1)$ smooth Weierstrass points [9].

Let $n$ be a positive integer. Let $\zeta$ denote a primitive $(2 n)$ th root of unity. Let $X$ denote the rational curve with $n+1$ nodes obtained from $\mathbf{P}_{\mathbf{C}}^{1}$ by identifying 0 with $\infty$, and $\zeta^{j}$ with $-\zeta^{j}$ for $j=0,1, \ldots, n-1$. Our main result is:

(0.1) Theorem. (1) If $n$ is even, then $X$ has no smooth Weierstrass points.

(2) If $n$ is odd, then $X$ has $2 n$ smooth Weierstrass points at the (2n)th roots of -1 .

In the first section, we prove a theorem that can be used to show that fixed points of an automorphism are frequently Weierstrass points, which extends known results for smooth curves. This is then used in the second section to prove the main result.

We thank Pierre Conner for very helpful discussions.

1

In this section, $X$ and $Y$ will denote integral projective curves over the complex numbers. If $C$ is a curve, we let $p_{a}(C)$ denote the arithmetic genus of $C$ and $p_{g}(C)$ denote the geometric genus of $C$. If $P \in C$, then we let $\tilde{\mathcal{O}}_{P}$ denote the normalization

Received by the editors September 14, 1994.

1991 Mathematics Subject Classification. Primary 14H55.

Key words and phrases. Weierstrass point, rational nodal curve.

(C)1996 American Mathematical Society 
of the local ring $\mathcal{O}_{P}=\mathcal{O}_{C, P}$, and we put $\delta_{P}$, the singularity degree of $P$, equal to $\operatorname{dim}_{\mathbf{C}} \tilde{\mathcal{O}}_{P} / \mathcal{O}_{P}$.

Let $\varphi: X \rightarrow Y$ be a finite morphism of degree $d$. Let $\alpha: \tilde{X} \rightarrow X$ and $\beta: \tilde{Y} \rightarrow Y$ denote the respective normalizations. We then have the following commutative diagram:

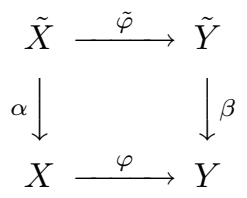

where $\tilde{\varphi}: \tilde{X} \rightarrow \tilde{Y}$ denotes the morphism induced by $\varphi$.

If we let

$$
R=\sum_{\tilde{P} \in \tilde{X}} r_{\tilde{P}} \tilde{P}
$$

denote the ramification divisor of the morphism $\tilde{\varphi}$, then the Riemann-Hurwitz formula gives the equation

$$
2 p_{g}(X)-2=d \cdot\left(2 p_{g}(Y)-2\right)+\operatorname{deg} R
$$

Now, we have

$$
\begin{aligned}
& p_{a}(X)=p_{g}(X)+\sum_{P \in X} \delta_{P} \\
& p_{a}(Y)=p_{g}(Y)+\sum_{Q \in Y} \delta_{Q} .
\end{aligned}
$$

Substituting into (1.1), we obtain

$$
2 p_{a}(X)-2=d \cdot\left(2 p_{a}(Y)-2\right)+\operatorname{deg} R+2 \cdot \sum_{P \in X} \delta_{P}-2 d \cdot \sum_{Q \in Y} \delta_{Q} .
$$

This formula may be considered a Riemann-Hurwitz formula for singular curves. Basically the same formula (for Gorenstein curves) appears in [12]. In the case when $Y$ is smooth, this formula appears in [3], and the term $\operatorname{deg} R+2 \cdot \sum_{P \in X} \delta_{P}$ may be viewed as the degree of the ramification class in Example 9.3.12 of [4]. Put

$$
r(\varphi)=\operatorname{deg} R+2 \cdot \sum_{P \in X} \delta_{P}-2 d \cdot \sum_{Q \in Y} \delta_{Q}
$$

We will now assume that $X$ is a Gorenstein curve. The classical theory of Weierstrass points on smooth curves was extended to Gorenstein curves in $[14,15,7]$. (For a further extension to integral curves, see [2].) We recall that if $p_{a}(X)$ is at least two, then every singular point of $X$ is a Weierstrass point of weight at least $\delta_{P}\left(p_{a}(X)-1\right) p_{a}(X)$. The theory at a smooth point of $X$ is entirely similar to the theory on a smooth curve; in particular, one may consider the gap sequence at a smooth point, and a smooth point is a Weierstrass point if and only if the gap sequence at that point is not $1,2, \ldots, p_{a}(X)$. The total of the weights of all Weierstrass points on $X$ is $p_{a}(X)^{3}-p_{a}(X)$.

The following theorem is an extension to the singular case of a result due to R.D.M. Accola [1]. 
(1.3) Theorem. Let $\varphi: X \rightarrow Y$ be a morphism of degree d. Let $P$ be a smooth point of $X$, and suppose $\varphi(P)$ is a smooth point of $Y$. If $r(\varphi)>4(d-1)$ and $r_{P}=d-1$ (i.e., $P$ is a point of total ramification), then $P$ is a Weierstrass point of $X$.

Proof. The proof is virtually the same as that of Accola [1], but we include it for the sake of completeness. Since $Q=\varphi(P)$ is a smooth point of $Y$, we may apply the Riemann-Roch Theorem for projective curves $[4,18.3 .4]$ to the line bundle $\mathcal{O}_{Y}\left(\left(p_{a}(Y)+1\right) Q\right)$ to infer that there exists a rational function $f \in k(Y)$ such that $\operatorname{ord}_{Q} f=-\left(p_{a}(Y)+1\right)$ and $f \in \mathcal{O}_{Y, Q^{\prime}}$ for all other points $Q^{\prime} \in Y$. Letting $\varphi^{*}: k(Y) \rightarrow k(X)$ denote the corresponding homomorphism of function fields, we see that $\varphi^{*}(f)$ will have order $-d\left(p_{a}(Y)+1\right)$ at $P$ (since $P$ is a point of total ramification) and will belong to $\mathcal{O}_{X, P^{\prime}}$ for all other points $P^{\prime} \in X$. Therefore, $P$ will be a Weierstrass point of $X$ if $d\left(p_{a}(Y)+1\right) \leq p_{a}(X)$. Now, this inequality will hold if and only if

$$
2 d\left(p_{a}(Y)+1\right)-2 \leq 2 p_{a}(X)-2=2 d\left(p_{a}(Y)-1\right)+r(\varphi),
$$

using equation (1.2). It follows from the definition of $r(\varphi)$ and equation (1.1) that $r(\varphi)$ is an even integer, so our hypothesis that $r(\varphi)>4(d-1)$ means that we have $r(\varphi) \geq 4 d-2$. Hence, the above inequalities are satisfied and $P$ is a Weierstrass point of $X$.

In the smooth case, Accola applied his theorem to deduce a well-known result of Lewittes [11]: if an automorphism of a smooth curve has at least five fixed points, then all the fixed points are Weierstrass points. In the next section, we will apply Theorem (1.3) to fixed points of an automorphism of a Gorenstein curve. We remark that the quotient of a Gorenstein curve by an automorphism need not be Gorenstein (see [13]).

If $X$ is singular and if $X /\langle\sigma\rangle$ is smooth, then one may sometimes conclude that every fixed point of $\sigma$ is a Weierstrass point of $X$ even when $\sigma$ has fewer than five fixed points, as in the following corollary.

(1.4) Corollary. Let $\varphi: X \rightarrow Y$ be a morphism from a Gorenstein curve $X$ to a smooth curve $Y$. Let $d$ denote the degree of $\varphi$ and put $N$ equal to the number of smooth totally ramified points on $X$. Put $\delta_{X}=\sum_{P \in X} \delta_{P}$. Then every smooth totally ramified point is a Weierstrass point of $X$ in each of the following cases:

(1) $N>4$ and $\delta_{X}=0$.

(2) $N>3$ and $\delta_{X}>0$.

(3) $N=3$ and $2 \delta_{X}>d-1$.

(4) $N=2$ and $\delta_{X}>d-1$.

(5) $N=1$ and $2 \delta_{X}>3(d-1)$.

Proof. The proof follows from Theorem (1.3) and the fact that

$$
r(\varphi) \geq N(d-1)+2 \delta_{X} .
$$

For a similar result in arbitrary characteristic, see [5]. 
(1.5) Corollary. Suppose $X$ is a Gorenstein curve with $\sum_{P \in X} \delta_{P}>1$. Suppose $\sigma$ is an involution of $X$ such that $X /\langle\sigma\rangle$ is smooth. Then every fixed point of $\sigma$ is a Weierstrass point of $X$.

Proof. Let $\varphi: X \rightarrow X /\langle\sigma\rangle$ denote the quotient map. Since $p_{a}(X)>1$, every singular point of $X$ is a Weierstrass point, so we may restrict our attention to smooth points of $X$. But every smooth fixed point is a Weierstrass point by Corollary (1.4).

\section{2}

Let $C$ denote the rational curve with $n+1$ nodes obtained from $\mathbf{P}_{\mathbf{C}}^{1}$ by identifying 0 with $\infty$, and $a_{j}$ with $-a_{j}$, where $\left\{a_{j},-a_{j}: j=1, \ldots, n\right\}$ is a set of $2 n$ distinct complex numbers. Let $P$ denote the node formed by identifying 0 with $\infty$.

Let $C^{\prime}$ denote the partial normalization of $C$ at $P$; that is, $C^{\prime}$ is the rational curve with $n$ nodes obtained from $\mathbf{P}_{\mathbf{C}}^{1}$ by identifying $a_{j}$ with $-a_{j}$ for $j=1, \ldots, n$. Then $C^{\prime}$ is a hyperelliptic curve with the hyperelliptic involution being given by $z \mapsto-z$. (The quotient of $C^{\prime}$ by this involution is simply $\mathbf{P}_{\mathbf{C}}^{1}$.) The smooth fixed points, namely 0 and $\infty$, are Weierstrass points on $C^{\prime}$, since at each of these points there is a rational function having a pole of order 2 and no other poles (or one can apply Corollary (1.5)). The gap sequence at each of these two points is $1,3, \ldots, 2 n-1$, and so the Weierstrass weight of each of these two points on $C^{\prime}$ is $(n-1) n / 2$. Each node on $C^{\prime}$ has Weierstrass weight $(n-1) n$, the minimum weight possible for a singular point on a curve with arithmetic genus $n$.

(2.1) Theorem. Let $P$ be an ordinary node on a Gorenstein curve $X$ of arithmetic genus $g$. Let $X^{\prime}$ denote the partial normalization of $X$ at $P$ and let $Q_{1}$ and $Q_{2}$ denote the points on $X^{\prime}$ lying over $P$. Then

$$
W_{X}(P)=(g-1) g+W_{X^{\prime}}\left(Q_{1}\right)+W_{X^{\prime}}\left(Q_{2}\right),
$$

where $W_{X}(P)$ (resp. $\left.W_{X^{\prime}}\left(Q_{i}\right)\right)$ denotes the Weierstrass weight of $P\left(\right.$ resp. $\left.Q_{i}\right)$ on $X\left(\right.$ resp. $\left.X^{\prime}\right)$.

Proof. [14]. (For a generalization of this result to arbitrary Gorenstein singularities see $[6,8]$.

(2.2) Corollary. Let $C$ denote the rational curve with $n+1$ nodes obtained from $\mathbf{P}_{\mathbf{C}}^{1}$ by identifying 0 with $\infty$, and $a_{j}$ with $-a_{j}$ for $j=1, \ldots, n$. Let $P$ denote the node formed by identifying 0 with $\infty$. Then the Weierstrass weight of $P$ on $X$ is $2 n^{2}$ and the number of smooth Weierstrass points on $C$ is at most equal to $2 n$.

Proof. The fact that the weight of $P$ is $2 n^{2}$ follows from Theorem (2.1) and the remarks preceding that Theorem. The number of smooth Weierstrass points on $C$ is at most

$$
n(n+1)(n+2)-2 n^{2}-n \cdot n(n+1)=2 n .
$$

Let $n$ be a positive integer. Let $\zeta$ denote a primitive $(2 n)$ th root of unity. Let $X$ denote the rational curve with $n+1$ nodes obtained from $\mathbf{P}_{\mathbf{C}}^{1}$ by identifying 0 with $\infty$, and $\zeta^{j}$ with $-\zeta^{j}$ for $j=0,1, \ldots, n-1$. Let $P$ denote the node formed by identifying 0 with $\infty$, and let $Q_{j}$, for $j=0,1, \ldots, n-1$, denote the node obtained by identifying $\zeta^{j}$ with $-\zeta^{j}$. By Corollary (2.2), the weight of $P$ is $2 n^{2}$. 
We will now give a proof of Theorem (0.1). Notice that Theorem (0.1) says that if $n$ is even, then $X$ has the minimum possible number (namely, zero) of smooth Weierstrass points, while if $n$ is odd, then $X$ has the maximum possible number of smooth Weierstrass points for a curve of the type considered in Corollary (2.2).

Proof of Theorem (0.1). (1) Assume that $n$ is even. Let $X_{0}$ denote the partial normalization of $X$ at the point $Q_{0}$. (Note that $Q_{0}$ is the node formed by identifying 1 and -1.) Then $X_{0}$ admits the involution $\sigma: z \mapsto 1 / z$. Let $Y$ denote the quotient of $X_{0}$ by this involution. Notice that the nodes $P$ and $Q_{n / 2}$ are fixed under $\sigma$, while the nodes $Q_{j}$ and $Q_{n-j}$ are mapped to each other by $\sigma$ for $j=1, \ldots,(n-2) / 2$. (The fact that $Q_{n / 2}$ is fixed under this involution is where we use the assumption that $n$ is even.) The quotient curve $Y$ then has $(n-2) / 2$ nodes corresponding to the orbits $\left\{Q_{j}, Q_{n-j}\right\}$, with all other points on $Y$ being smooth. (Note that the images of $P$ and $Q_{n / 2}$ on $Y$ are nonsingular points since the involution glues together the two branches at each of these nodes.)

We claim that the points 1 and -1 are Weierstrass points on $X_{0}$. To see this, apply Theorem (1.3) to the quotient map

$$
\varphi: X_{0} \rightarrow Y=X_{0} /\langle\sigma\rangle
$$

The points 1 and -1 are fixed points of $\sigma$, hence total ramification points of $\varphi$. The induced map $\tilde{\varphi}: \mathbf{P}_{\mathbf{C}}^{1} \rightarrow \mathbf{P}_{\mathbf{C}}^{1}$ is such that the target $\mathbf{P}_{\mathbf{C}}^{1}$ is the quotient of the source $\mathbf{P}_{\mathbf{C}}^{1}$ by $\tilde{\sigma}$, the lifting of $\sigma$ to the normalization of $X_{0}$. Thus, we have $\operatorname{deg} R=2$. Since $X_{0}$ has $n$ nodes and $Y$ has $(n-2) / 2$ nodes, we have

$$
r(\varphi)=2+2 n-4(n-2) / 2=6
$$

It follows from Theorem (1.3) that 1 and -1 are Weierstrass points of $X_{0}$.

Now, we see, from Theorem $(2.1)$, that $W_{X}\left(Q_{0}\right)$, the Weierstrass weight of $Q_{0}$ on $X$, is at least $n^{2}+n+2$. For $k=1, \ldots, n-1$, the map $\tau_{k}: z \mapsto \zeta^{k} z$ on the extended complex plane induces an automorphism of $X$ that takes $Q_{j}$ to $Q_{j+k \bmod n}$, $j=0,1, \ldots, n-1$. It follows that the Weierstrass weight of $Q_{j}$, for $j=1, \ldots, n-1$, is the same as the Weierstrass weight of $Q_{0}$. Thus the sum of the Weierstrass weights of the nodes on $X$ is

$$
\begin{aligned}
W_{X}(P)+\sum_{j=0}^{n-1} W_{X}\left(Q_{j}\right) & \geq 2 n^{2}+n\left(n^{2}+n+2\right) \\
& =n^{3}+3 n^{2}+2 n \\
& =n(n+1)(n+2) \\
& =p_{a}(X)^{3}-p_{a}(X) .
\end{aligned}
$$

Since this is the total of the weights of all Weierstrass points on $X$, the above inequality is an equality and there are no smooth Weierstrass points on $X$.

(2) Assume that $n$ is odd. Consider the involution $\sigma^{\prime}: z \mapsto-1 / z$ on $X$. The nodes $P$ and $Q_{0}$ are fixed under $\sigma^{\prime}$, while the nodes $Q_{j}$ and $Q_{n-j}$ are mapped to each other for $j=1, \ldots,(n-1) / 2$. Let $Y^{\prime}$ denote the quotient of $X$ by $\sigma^{\prime}$. Then 
$Y^{\prime}$ has $(n-1) / 2$ nodes. Applying Theorem (1.3), as in the previous case, we see that $i$ and $-i$, which are fixed points of $\sigma^{\prime}$, are Weierstrass points of $X$. Since the automorphisms $\tau_{k}: z \mapsto \zeta^{k} z$ map $i$ to all the other $(2 n)$ th roots of -1 , we see that each of these $2 n$ points is a Weierstrass point of $X$.

Let $P_{1}, \ldots, P_{2 n}$ denote the $(2 n)$ th roots of -1 . Then we have

$$
\begin{aligned}
W_{X}(P)+\sum_{j=0}^{n-1} W_{X}\left(Q_{j}\right)+\sum_{k=1}^{2 n} P_{k} & \geq 2 n^{2}+n \cdot n(n+1)+2 n \\
& =n^{3}+3 n^{2}+2 n=n(n+1)(n+2) \\
& =p_{a}(X)^{3}-p_{a}(X) .
\end{aligned}
$$

Therefore, each of the $(2 n)$ th roots of -1 is a smooth Weierstrass point of weight 1 , and each of the nodes $Q_{j}, j=0, \ldots, n-1$, has (minimal) weight $n(n+1)$ on $X$. $\square$

It seems plausible that any rational nodal curve with no smooth Weierstrass points is isomorphic to one of the curves in case (1) in Theorem (0.1); in particular, this would mean that a rational nodal curve with an even number of nodes would have to have at least one smooth Weierstrass point. We note that a rational nodal curve with two nodes always has two smooth Weierstrass points, and any rational nodal curve with three nodes and no smooth Weierstrass points is isomorphic to Widland's example [10].

\section{REFERENCES}

1. R.D.M. Accola, On generalized Weierstrass points on Riemann surfaces, Modular functions in analysis and number theory, Lecture Notes Math. Stat., University of Pittsburgh, Pittsburgh, PA, 1978, pp. 1-19. MR 85f:14016

2. E. Ballico and L. Gatto, Weierstrass points on singular curves, preprint.

3. N. Chiarli, A Hurwitz type formula for singular curves, C. R. Math. Rep. Acad. Sci. Canada 6 (1984), 67-72. MR 85e:14037

4. W. Fulton, Intersection Theory, Springer, Berlin-Heidelberg-New York, 1984. MR 85k:14004

5. A. Garcia, On Weierstrass points on Artin-Schreier extensions of $k(x)$, Math. Nachr. 144 (1989), 233-239. MR 91f:14021

6. A. Garcia and R.F. Lax, Weierstrass weight of Gorenstein singularities with one or two branches, Manuscripta Math. 81 (1993), 361-378. MR 94j:14032

7. A. Garcia and R.F. Lax, Weierstrass points on Gorenstein curves in arbitrary characteristic, Comm. Algebra 22 (1994), 4841-4854. MR 95f: 14063

8. A. Garcia and R.F. Lax, On canonical ideals, intersection numbers, and Weierstrass points on Gorenstein curves, J. Alg. (to appear).

9. R. F. Lax, Weierstrass points on rational nodal curves, Glasgow Math. J. 29 (1987), 131-140. MR 88a:14016

10. R.F. Lax and C. Widland, Weierstrass points on rational nodal curves of genus 3, Canad. Math. Bull. 30 (1987), 286-294. MR 88h:14039

11. J. Lewittes, Automorphisms of compact Riemann surfaces, Amer. J. Math. 85 (1963), 732752. MR 28:4102

12. A. Oneto And E. Zatini, Finite morphisms of Gorenstein curves, Commutative algebra (Trento, 1981), Lecture Notes in Pure and Appl. Math. No. 84, Dekker, New York, 1983 pp. 197-210. MR 84g:14051

13. K. Watanabe, Certain invariant subrings are Gorenstein II, Osaka J. Math. 11 (1974), 379388. MR 50:7124 
14. C. Widland, Weierstrass points on Gorenstein curves, Ph.D. dissertation, Louisiana State University, 1984

15. C. Widland and R.F. Lax, Weierstrass points on Gorenstein curves, Pac. J. Math. 142 (1990), 197-208. MR 91b:14037

IMPA, Estrada Dona Castorina 110, 22.460 Rio de Janeiro, Brasil

E-mail address: garcia@impa.br

Department of Mathematics, Louisiana State University, Baton Rouge, Louisiana 70803

E-mail address: lax@math.lsu.edu 